\title{
Le financement public des cultes en France entre régime d'exception et droit commun
}

Pierre-Henri Prélot

\section{OpenEdition}

\section{Journals}

Édition électronique

URL : http://journals.openedition.org/rdr/1037

DOI : $10.4000 /$ rdr. 1037

ISSN : 2534-7462

Éditeur

Presses universitaires de Strasbourg

Édition imprimée

Date de publication : 10 mai 2016

Pagination : $55-66$

ISBN : 978-2-86820-954-2

ISSN : 2493-8637

\section{Référence électronique}

Pierre-Henri Prélot, «Le financement public des cultes en France entre régime d'exception et droit commun », Revue du droit des religions [En ligne], 1 | 2016, mis en ligne le 12 février 2020, consulté le 19 novembre 2020. URL : http://journals.openedition.org/rdr/1037 ; DOI : https://doi.org/10.4000/rdr. 1037

La revue du droit des religions est mise à disposition selon les termes de la Creative Commons Attribution - Pas d'Utilisation Commerciale 4.0 International - CC BY-NC 4.0. 


\section{LE FINANCEMENT PUBLIC DES CULTES EN FRANCE ENTRE RÉGIME D'EXCEPTION ET DROIT COMMUN}

\section{Pierre-Henri PRÉLOT}

Université de Cergy-Pontoise

\section{RésuMÉ}

Le droit du financement public des activités religieuses en France s'est longtemps défini par son statut d'exception. C'était un droit d'exception au regard des autres activités sociales, dans la mesure où la loi du 9 décembre 1905 consacre en son article 2 un principe spécifique d'interdiction de toute subvention en faveur des cultes. C'était un droit d'exception au sens territorial également, dans la mesure où le droit « commun » des activités religieuses que définit la loi de 1905 ne s'applique pas en certaines parties du territoire. Cette double exceptionnalité est en passe de s'estomper, au profit d'un régime de financement public des activités religieuses qui tend à se rapprocher du droit commun du financement des autres activités sociales, et ce dans toutes les parties du territoire.

\section{ABSTRACT}

Public funding of religious activities in France has been characterized for a long time by its exceptional status. It establishes an exception in relation to other social activities, insofar as Article 2 of the 1905 Act enshrines a specific principle of prohibition of any public financial support to religious groups. It was a law of exception in the territorial sense too, since the common law of religious activities defined by the 1905 Act does not apply in certain geographical areas. This double exceptionality is about to fade, in favour of a public funding of religious activities which tends to be closer to the common law of funding of social activities, and in all parts of the territory. 


\section{L'INTERDICTION DE FINANCER LES CULTES, PRINCIPE DE DROIT COMMUN RELATIF}

La loi de 1905 supprime le régime des cultes reconnus mis en place au début du XIX ${ }^{e}$ siècle qui reposait sur des mécanismes de reconnaissance différenciés : concordat et articles organiques pour la religion catholique, articles organiques des cultes protestants pour la religion luthérienne et la religion réformée (loi du 18 germinal an X), décret du 17 mars 1808, loi du 8 février 1831 et ordonnance du 25 mai 1844 pour la religion juive. Désormais, les religions seront soumises à un même régime légal qui instaure ce que l'on peut appeler un droit commun des activités religieuses. Les inclinations libérales de la loi de 1905 tendent par ailleurs à rapprocher ce droit commun des activités religieuses du droit commun des autres activités sociales. L'association cultuelle qui constitue la forme légale d'organisation des religions dans le régime de séparation est ainsi reprise du titre premier de la loi de 1901 sur la liberté des associations, et non de son titre III qui organise une procédure spécifique de reconnaissance pour les congrégations religieuses. Le régime des processions religieuses sur la voie publique est quant à lui fixé par renvoi pur et simple à la loi municipale de 1884 (article 27) et relève ainsi du droit commun des manifestations publiques. Il en va de même pour les réunions de fidèles, à propos desquelles le législateur de 1907 renvoie à la loi de 1881 sur la liberté de réunion. Et malgré l'existence dans la loi d'un titre V consacré à la «police des cultes », il est manifeste en parcourant les articles qu'il contient que, pour l'essentiel, la police des activités religieuses en régime de séparation n'est pas autre chose que la police de l'ordre public appliquée aux cultes.

Mais ce libéralisme n'est pas univoque, et la loi de 1905 contient aussi des dispositions qui contredisent cette tendance à la banalisation juridique des activités religieuses. C'est le cas en particulier de tout ce qui concerne leur financement, dont le régime est partagé entre droit commun et régime d'exception. Désormais, les religions sont tenues de pourvoir elles-mêmes à leur propre entretien et, comme on le sait, c'est la loi de 1905 qui est au point de départ de la création par l'Église catholique du denier du culte qui assure depuis la séparation l'essentiel de son financement. Mais dans le même temps, le législateur restreint considérablement, dans un but de contrôle, la faculté qu'ont les associations cultuelles de faire usage des fonds qu'elles recueillent. En cela les « cultuelles » de la loi de 1905 diffèrent profondément de leurs homologues de la loi de 1901. De la même façon, la loi supprime logiquement le budget des cultes qui assurait la rémunération des personnels religieux, mais 
elle va beaucoup plus loin en interdisant à l'avenir toute possibilité, même ponctuelle, de financement public des activités religieuses quelles qu'elles soient. Les religions se trouvent ainsi soumises à un régime d'exception qui les distingue des autres activités sociales, à l'encontre desquelles ne pèse à l'époque aucun interdit analogue ${ }^{1}$. Le but poursuivi est alors d'interdire aux communes de reconstituer à leur échelon le budget des cultes.

Mais le propre d'un régime d'interdiction, surtout lorsqu'il est aussi strict, est généralement de se voir allégé au fil du temps par des dérogations ${ }^{2}$ destinées à autoriser ce que la rigidité de la règle ne permet pas. Les dérogations légales se sont ainsi accumulées au fil du temps, à commencer par l'exception relative aux aumôneries des services publics, qui figurait dans la loi de séparation elle-même $e^{3}$. On ne reprendra pas ici l'énumération des nombreuses dispositions législatives adoptées depuis cette époque, et dont la dernière en date consiste dans la consolidation juridique des baux emphytéotiques administratifs, dits BEA « cultuels », dans le Code général des collectivités territoriales en $2006^{4}$. Toutes ces dérogations n'ont bien entendu pas remis en cause le principe même de l'interdiction des subventions aux cultes, mais elles en ont singulièrement rétréci le champ. Ce sont d'ailleurs ces dérogations qui ont inspiré en réaction la revendication d'une constitutionnalisation du titre premier de la loi de 1905, en tant que composante substantielle du principe de laïcité ${ }^{5}$.

L'interdiction de subventionner les cultes définit ce qu'on peut appeler le droit commun financier de la séparation des Églises et de l'État. Toutefois ce droit commun séparatiste ne s'applique pas sur l'ensemble du territoire français. Un décret du 6 février 1911 étend l'application de la loi de 1905 à la

1. Il existe à l'époque une autre exception, qui est celle de l'enseignement privé, mais le fondement de l'interdiction est rigoureusement identique, à savoir que l'enseignement privé est pris en charge par l'Église catholique.

2. On retrouve également des dérogations en matière d'enseignement privé, par exemple à l'article 69 (maintenu) de la loi Falloux du 15 mars 1850.

3. Peu de temps après, la loi du 13 avril 1908 autorisait les collectivités propriétaires à engager les dépenses nécessaires pour l'entretien et la conservation des édifices affectés au culte, ici encore en contradiction avec le principe d'interdiction de l'article 2.

4. La mise à disposition de terrains par une collectivité publique, en vue de la construction d'un édifice cultuel, par la voie d'un bail emphytéotique administratif (BEA) à objet cultuel est une pratique remontant à 1931, avec les fameux Chantiers du Cardinal. Les BEA à objet cultuel sont désormais mentionnés expressément à l'article L. 1311-2 du Code général des collectivités territoriales (CGCT) en 2006. Le Conseil d'État a considéré (CE Ass. 19 juill. 2011, n 320796 Mme Vayssière) qu'avec l'article L. 1311-2 le législateur «a, ce faisant, dérogé » à la loi de 1905.

5. Proposition $n^{\circ} 46$ du candidat François Hollande à l'élection présidentielle de 2012. 
Martinique, à la Guadeloupe et à la Réunion. Mais il ne s'agit alors que d'une toute petite partie de l'espace colonial. Par ailleurs, au retour à la France de l'Alsace et de la Moselle en 1919, il est décidé de maintenir dans les trois départements de l'Est le système des cultes reconnus qui est, du point de vue du financement, l'antithèse absolue du nouveau régime de séparation. Non seulement un certain nombre de dépenses y ont un caractère obligatoire - c'est le cas de la rémunération des ministres des cultes reconnus - mais, de surcroît, toutes les autres dépenses en faveur des cultes restent autorisées. Ce sont des mécanismes apparentés qui s'appliquent en Guyane en vertu de l'ordonnance royale du 27 août 1828. Mais là-bas, seuls les ministres du culte catholique bénéficient d'une rémunération publique. Enfin, dans les autres territoires d'outre-mer, où la loi de 1905 n'a pas été introduite, et où s'appliquent les décrets Mandel de $1939^{6}$, le clergé local ne fait pas l'objet d'une prise en charge (exception faite de Saint-Pierre et Miquelon), mais le financement public des activités religieuses reste également autorisé à la différence du territoire métropolitain. Tous ces régimes locaux présentent donc une caractéristique commune, à savoir que le financement des activités religieuses n'y est pas prohibé dans son principe. En cela, ils se rapprochent du droit commun des autres activités sociales, que les pouvoirs publics sont en droit de soutenir financièrement dès lors qu'elles ne sont pas l'objet d'une interdiction analogue à celle de l'article 2. Le sens qui doit être donné à cette absence d'interdiction de financer les cultes, c'est que les activités religieuses, au même titre que les activités sportives, artistiques ou culturelles, ne sont pas par principe exclusives de tout caractère d'intérêt public. Mais dans le régime de reconnaissance où les cultes étaient compris comme des services publics, cet intérêt public était présumé, ce qui, comme on le verra, n'est plus le cas aujourd'hui.

\section{LA REMISE EN CAUSE DES FINANCEMENTS OBLIGATOIRES}

Ce qui dans les régimes locaux retient le plus l'attention, plus encore que l'absence de toute interdiction de financer les cultes, ce sont bien entendu les mécanismes de financement obligatoire et en particulier la rémunération des ministres du culte en Alsace-Moselle et en Guyane. Ces mécanismes ont survécu, depuis un siècle, aux nombreuses remises en cause politiques dont

6. Polynésie française, Wallis et Futuna, Saint-Pierre et Miquelon, Terres australes et antarctiques françaises, Nouvelle-Calédonie et Mayotte. Décret du 16 janvier 1939 instituant outre-mer des conseils d'administration des missions religieuses (modifié par décret du 6 décembre 1939). 
ils ont été l'objet, à tel point qu'ils semblent figés pour l'éternité. Pourtant à y regarder de près, ces régimes sont aujourd'hui extrêmement fragilisés, et cette fragilité pourrait bien être le prélude à leur suppression définitive même si leur remise en cause n'est pas encore à l'ordre du jour. En ce qui concerne l'Alsace-Moselle tout d'abord, le Conseil constitutionnel a jugé dans sa décision QPC du 21 février 2013 Association pour la promotion et l'expansion de la laïcité, que le salariat des ministres du culte était contraire au principe constitutionnel de laïcité ${ }^{7}$. Mais en ajoutant dans cette même décision que le législateur constituant n'avait pas entendu pour autant, en 1946 et en 1958, abroger ce régime ${ }^{8}$, il l'a maintenu en vigueur à titre dérogatoire, en renvoyant au législateur le soin de prendre le moment venu la décision politique d'y mettre fin. Il ne faut pas s'y tromper toutefois, montrer une direction c'est inviter à la prendre, et la voie indiquée par l'aiguilleur finira par être empruntée par le train un jour ou l'autre.

Dans l'état actuel des choses, l'inconstitutionnalité du salariat des ministres du culte exclut toute possibilité d'extension de la reconnaissance à de nouveaux cultes, et en particulier à l'islam. De surcrô̂t, la situation constitutionnelle particulière du droit local, dont le Conseil constitutionnel a jugé qu'il ne pouvait faire l'objet d'aménagements « que dans la mesure où les différences de traitement qui en résultent ne sont pas accrues et que leur champ d'application n'est pas élargi » appelle une convergence progressive de celui-ci vers le droit commun de la laïcité ${ }^{9}$, pour autant qu'il doive évoluer de quelque façon que ce soit. Cette impossibilité qu'il y a désormais de réformer le droit local dans le sens d'une égalité de traitement revient à le condamner en tant que modèle alternatif de régulation publique des religions.

7. Cons. const., déc. 21 févr. 2013, n 2012-297 QPC, Association pour la promotion et l'expansion de la laïcité. Dans le considérant $\mathrm{n}^{\circ} 5$ de la décision, le Conseil constitutionnel énonce que « le principe de laïcité [...] implique que celle-ci [la République] ne salarie aucun culte». L'affirmation selon laquelle «La France est une République indivisible, laique, démocratique et sociale » figure dans la Constitution depuis 1946.

8. "Considérant, toutefois, qu'il ressort tant des travaux préparatoires du projet de la Constitution du 27 octobre 1946 relatifs à son article ler que de ceux du projet de la Constitution du 4 octobre 1958 qui a repris la même disposition, qu'en proclamant que la France est une «République... laïque», la Constitution n'a pas pour autant entendu remettre en cause les dispositions législatives ou réglementaires particulières applicables dans plusieurs parties du territoire de la République lors de l'entrée en vigueur de la Constitution et relatives à l'organisation de certains cultes et, notamment, à la rémunération de ministres du culte " (Considérant n ${ }^{\circ}$ ).

9. Cons. const., déc. 5 août 2011, n 2011-157 QPC, Société SOMODIA. Les décisions ultérieures ont montré que le Conseil constitutionnel faisait une application extrêmement stricte de ce principe de convergence. 
C'est cette voie de la réforme qu'a choisie quant à lui le Luxembourg, qui a su faire évoluer en profondeur, à partir de 2015, son régime des cultes également hérité en large part du système napoléonien. Ce modèle de reconnaissance fondé sur la rémunération publique des ministres du culte correspond en fait au contexte d'une société fortement cléricalisée, où les autorités religieuses sont investies d'une fonction d'encadrement des populations. Il n'est plus du tout en cohérence avec la réalité des sociétés modernes sécularisées, dans lesquelles les religions ont perdu cette fonction traditionnelle de contrôle social, et où le soutien public dont elles bénéficient ne peut se justifier le cas échéant que par la volonté de donner toute son effectivité à la liberté religieuse, pour cette partie de la population qui y reste attachée. De surcroît, dans des périodes de repli économique, il devient plus difficile aux populations de comprendre que les autorités religieuses continuent à être entretenues aux frais de l'État. Tous ces arguments qui ont conduit à la réforme du droit luxembourgeois valent peu ou prou pour l'Alsace-Moselle.

La situation de la Guyane est, à certains égards, assez proche de celle qui prévaut en Alsace-Moselle. Mais à la différence du régime des cultes reconnus de métropole, l'ordonnance du roi concernant le gouvernement de la Guyane française, en date du 27 août 1828, prescrit la prise en charge financière du seul clergé catholique, soit aujourd'hui l'évêque de Cayenne et une trentaine de prêtres. Invoquant les difficultés financières de sa collectivité, le président du conseil général de Guyane a refusé en $2014^{10}$ d'inscrire au budget départemental les sommes nécessaires, mais le tribunal administratif de Cayenne saisi d'un recours a considéré qu'il s'agit d'une dépense légale dont la collectivité ne peut s'affranchir elle-même ${ }^{11}$.

Dans la décision QPC du 21 février 2013, le Conseil constitutionnel a jugé contraire au principe constitutionnel de laïcité le seul salariat des ministres du culte, mais en réalité c'est bien le régime financier des cultes reconnus qui est remis en cause dans son ensemble. L'obligation faite aux communes ${ }^{12}$ d'assurer le logement des ministres du culte s'analyse en effet comme une prestation en nature et, compte tenu de son caractère obligatoire, elle est une composante du salariat au sens de la législation des cultes. C'est d'ailleurs pour empêcher la prolongation, sur une base volontaire, de la prise en charge

10. La loi de finances pour 1901 a transféré la prise en charge des cultes de l'État à la colonie, en sorte que c'est aujourd'hui sur la collectivité départementale qu'elle repose.

11. TA Cayenne, 29 déc. 2014, n 1400708.

12. Art. 92 du décret du 30 décembre 1809 pour les ministres du culte catholique, ordonnance du 7 août 1842 pour les ministres protestants et israélites. CGCT, art. L. 2543-3 2. 
publique des ministres du culte par les communes que la loi du 2 janvier $1907^{13}$ prescrivait que la location des presbytères devait être approuvée par l'administration préfectorale. L'autre volet du financement contraint des cultes reconnus tient dans l'obligation faite aux communes, en vertu du principe de subsidiarité, de combler le cas échéant le déficit des établissements publics des cultes reconnus (fabriques, conseils presbytéraux et consistoires) ${ }^{14}$. Les établissements publics du culte n'étant fondés à engager, en application du principe de spécialité, que des dépenses relatives au culte, c'est essentiellement à raison de travaux importants sur les édifices que les communes seront tenues d'intervenir. Mais ici, le droit local tend en réalité à se rapprocher du droit général, dans la mesure où les communes de vieille France, même si rien ne les y contraint, se font généralement une obligation d'entretenir elles aussi les édifices légalement affectés au culte dont elles ont la propriété, et qu'elles sont par ailleurs fondées à financer les travaux de réparation des édifices du culte appartenant aux associations cultuelles en application de la loi du 25 décembre 1942.

Quant aux dépenses relatives à l'exercice du culte qui ne présentent pas de caractère obligatoire, elles peuvent être prises en charge par les communes. Si comme a pu le dire le Conseil constitutionnel dans sa décision précitée de 2013, le principe de laïcité implique que la République " ne salarie aucun culte ", il ne s'oppose pas en revanche à ce qu'elle les subventionne. Ici, c'est l'article 2 de la loi de 1905 qui marque la différence entre le régime de laïcité et les territoires où la loi de séparation n'a pas été introduite. Mais comme il reste à l'expliquer, ces deux régimes tendent également à se rapprocher de plus en plus.

\section{L'INTÉRÊT GÉNÉRAL COMME FONDEMENT DU SOUTIEN FINANCIER AUX RELIGIONS}

En consacrant le caractère simplement législatif de l'interdiction du financement public des cultes, la décision du Conseil constitutionnel Association pour la promotion et l'expansion de la laïcité a eu pour effet de consolider la jurisprudence récente du Conseil d'État autorisant désormais, sous certaines conditions qu'il précise, le versement de subventions aux cultes, et qui prend

13. Loi du 2 janvier 1907, art. $1^{\text {er }}$, al. 3 .

14. CGCT, art. L. 2543-3 3․ 
son point de départ dans l'arrêt Ministre de l'Outre-mer de $2005^{15}$. Cet arrêt fondamental autorise le versement par le président du territoire de la Polynésie française d'une subvention d'équipement à l'Église évangélique locale, en vue de financer la reconstruction d'un presbytère détruit par un cyclone. Ainsi que le relèvent les juges du fond, « ce bâtiment jouait un rôle dans de nombreuses activités socio-éducatives notamment dans les îles éloignées comme l'île de Raiatea » et de surcroît « lors du passage des cyclones, le presbytère est ouvert à tous et accueille les sinistrés ». L'intérêt particulier de cet arrêt, du point de vue du droit de la laïcité, réside dans le fait qu'aux yeux des juges l'absence d'application de l'article 2 n'est pas en soi suffisante pour autoriser le versement de subventions à une religion. La subvention publique doit être justifiée en tout état de cause par un intérêt public local qu'elle vise à satisfaire, en l'espèce l'accueil des populations lors des cyclones. Autrement dit, l'intérêt religieux n'est pas en soi constitutif d'un intérêt public.

Cette jurisprudence a fait l'objet d'une transposition quasi immédiate à l'Alsace-Moselle, à propos du versement par une commune d'une subvention de 1500 euros à une association de droit local, destinée à couvrir partiellement les dépenses d'un pèlerinage à Rome organisé au bénéfice des servants d'autel. Reprenant quasiment mot pour mot la formulation du Conseil d'État de 2005, la cour administrative d'appel de Nancy énonce dans son arrêt Commune de Soultz de $2008{ }^{16}$ que « si le principe constitutionnel de laïcité, qui s'applique sur l'ensemble du territoire de la République et implique la neutralité de l'État et des collectivités territoriales et un traitement égal des différents cultes, n'interdit pas, par lui-même, l'octroi par une commune de subventions à des activités dépendant des cultes, l'attribution de telles subventions est subordonnée à l'existence d'un intérêt général ». Elle en déduit que « s'il est certain que le pèlerinage accompli à Rome comportait également un aspect culturel, la dimension religieuse de ce voyage apparaît prépondérante tant dans son principe même que dans son déroulement ", et " qu'ainsi [...] l'objectif confessionnel essentiellement poursuivi par les organisateurs doit faire regarder en l'espèce l'octroi de la subvention litigieuse comme ne répondant pas à un intérêt général pour la commune ». Il y a dans la part respective accordée par les juges à la dimension culturelle et à la dimension religieuse du voyage la place pour la discussion, et l'on peut légitimement estimer qu'au point de vue culturel, le déplacement en Italie d'un groupe de servants de messe présente autant d'intérêt que celui de n'importe quel autre

15. CE, 16 mars 2005, $\mathrm{n}^{\circ}$ 265560, Ministre de l'Outre-mer.

16. CAA Nancy, 6 mars 2008, n 07NC00083, Commune de Soultz. 
groupe d'administrés dépourvus de motivation religieuse. Mais là n'est pas la question. Ce que cet arrêt met en lumière, c'est que même lorsqu'il existe un régime légal de financement des cultes, comme c'est le cas en Alsace-Moselle, la prise en charge des activités religieuses sur une base volontaire n'est pas autorisée pour autant. Autrement dit, si les collectivités publiques peuvent financer les religions, ce ne peut être que pour un motif d'intérêt général distinct de l'intérêt religieux lui-même.

En faisant prévaloir le critère de l'intérêt public sur le principe historique de la liberté des subventions aux cultes, l'arrêt Ministre de l'Outre-mer laissait supposer que ce même critère de l'intérêt public pourrait prévaloir y compris en régime d'interdiction. C'est ce que viennent établir les cinq arrêts du Conseil d'État en date du 19 juillet $2011^{17}$, validant dans un contexte à chaque fois différent les subventions accordées par des collectivités publiques à des projets présentant tout à la fois un intérêt religieux et un intérêt public spécifique. Ces solutions ont été abondamment confirmées, et elles marquent un tournant important dans la jurisprudence du Conseil d'État. D'un point de vue purement formel, la Haute juridiction a également abandonné l'obligation qu'elle avait précédemment imposée aux groupements religieux de dissocier leurs activités à caractère religieux et celles à caractère profane dans des structures associatives spécifiques ${ }^{18}$.

On ne fera pas l'exposé de détail de tous les arrêts rendus par le Conseil d'État depuis 2011, et qui confirment la légalité de financements publics présentant un caractère d'intérêt général spécifique ${ }^{19}$, détachable de l'avantage financier qu'ils confèrent à une religion. Ce qui retient surtout l'attention, c'est qu'il n'y a pas de différence de fond entre ces arrêts de 2011 et celui de 2005 rendu à propos de l'outre-mer, en sorte que l'application ou la non-application de la loi de 1905 ne change rien quant au régime des subventions. À la

17. CE 19 juill. 2011, n 320796, Dame Vayssière; CE, 19 juill. 2011, n 313518, Commune de Montpellier; CE, 19 juill. 2011, n 309161, Communauté urbaine du Mans - Le Mans métropole; CE, 19 juill. 2011, n 308544, Commune de Trélazé; CE, 19 juill. 2011, $\mathrm{n}^{\circ}$ 308817, Fédération de la libre pensée et de l'action sociale du Rhône et M. P.

18. CE, 4 mai 2012, n 336462, Fédération de la libre pensée et d'action sociale du Rhône, à propos d'une subvention à la communauté de San Egidio. La décision revient sur la jurisprudence Association Siva Soupramanien de Saint-Louis de 1992 (CE, 9 oct. 1992, $\mathrm{n}^{\circ} 94455$.

19. Dans l'arrêt ADEME du 26 novembre 2012 (n 344379), le Conseil d'État souligne ainsi qu'un établissement public « ne peut accorder une subvention à une association qu'en vue de la réalisation d'un projet, d'une manifestation ou d'une activité qui ne présente pas un caractère cultuel et n'est pas destiné au culte et à la condition [...] que le soutien de ce projet, cette manifestation ou cette activité s'inscrive dans le cadre des missions d'intérêt général qui lui ont été confiées par le législateur ». 
lumière des arrêts de 2011, il est clair que le Conseil d'État aurait pu rendre la même décision en 2005 si l'article 2 s'était appliqué en Polynésie. Autrement dit, le régime de séparation et les régimes locaux dérogatoires tendent à se rapprocher du point de vue du financement des cultes, et c'est l'existence résiduelle en Alsace-Moselle et en Guyane d'un régime de salariat qui fait entre eux la différence essentielle. Et si l'appréciation concrète du caractère d'intérêt public d'un financement devait varier d'un lieu à l'autre, ce qui on l'a dit n'apparaît pas actuellement dans la jurisprudence du Conseil d'État, c'est essentiellement au regard de l'avantage dont jouissent les religions bénéficiant d'un financement légal, dans une logique de compensation au bénéfice des autres cultes et notamment de l'islam en situation de désavantage. C'est ainsi que les collectivités territoriales alsaciennes, et notamment la ville de Strasbourg, ont financé partiellement la construction de la grande mosquée, inaugurée en 2012, alors qu'il leur aurait été plus difficile de le faire en régime de séparation ${ }^{20}$.

\section{CONCLUSION}

Depuis les temps les plus anciens, l'encadrement juridique des activités religieuses a toujours été en France une matière hautement politique, puisqu'en délimitant la place accordée aux religions dans l'espace social, c'est sa propre souveraineté temporelle que le pouvoir affirmait et protégeait tout à la fois. Pour cette raison, le droit des activités religieuses s'est construit et transformé sur un mode essentiellement écrit, que ce soit selon le modèle classique des concordats, sous une forme quasi constitutionnelle (Constitution civile du clergé), et pour finir par des lois (articles organiques du Concordat, et aujourd'hui loi de séparation). C'est cette vision politique héritée de l'histoire qui a conduit à la sacralisation progressive de la loi de 1905, en tant que garantie fondamentale de la souveraineté laĩque de l'État. Et c'est cette même vision politique qui est au fondement de lois telles que celle de 2004, interdisant le port de signes ostensibles d'appartenance religieuse dans l'école publique, et celle de 2010 interdisant la dissimulation du visage dans l'espace

20. Dans son arrêt Communauté urbaine du Mans du 19 juillet 2011, le Conseil d'État a toutefois estimé, à propos de la construction sur fonds publics d'un abattoir destiné à l'abattage rituel, que l'intérêt public pouvait tenir "notamment à la nécessité que les cultes soient exercés dans des conditions conformes aux impératifs de l'ordre public ». Un tel énoncé pourrait concerner la construction de lieux de culte dans les lieux où il existe une demande forte, ce qui était précisément le cas à Strasbourg. 
public. Mais en affirmant la liberté de religion dans sa double dimension individuelle et collective ${ }^{21}$, la loi de 1905 opérait une rupture décisive, à savoir que la religion cessait d'être une affaire de pure souveraineté comme elle l'avait toujours été pour le pouvoir, mais qu'elle devenait désormais aussi une question de liberté individuelle, protégée à ce titre par le droit et par les institutions de l'État.

La principale manifestation de ce changement de paradigme, c'est la montée en puissance progressive, tout au long du $\mathrm{Xx}^{\mathrm{e}}$ siècle, de la protection juridictionnelle des convictions et des pratiques religieuses, aboutissant à l'édification, à côté des sources législatives et administratives classiques formalisées dans des textes écrits, d'un droit de la liberté de religion moins directement politique, et de plus en plus jurisprudentiel. Peu à peu la liberté de religion est pleinement devenue, au même titre que les autres libertés publiques et privées, un droit individuel subjectif protégé par le juge national, sous le contrôle de plus en plus exigeant de la Cour européenne des droits de l'homme. C'est ainsi que le principe objectif de laïcité de l'État républicain est lui-même à ranger depuis 2013 au nombre de ces « droits et libertés que la Constitution garantit $»^{22}$, dont chacun est fondé à se prévaloir devant le juge ${ }^{23}$.

Cette évolution du droit écrit de la laïcité et de la liberté de religion vers un droit de plus en plus jurisprudentiel ne pouvait pas rester sans conséquence sur la substance même de ce droit. Elle a ainsi permis d'accompagner les transformations considérables que les mutations de la sociologie religieuse ont fait subir au fil du temps à l'application du texte de $1905^{24}$, qui comme n'importe quel texte juridique a dû être appliqué à la lumière des conditions du temps présent, afin de le préserver de la désuétude. Ainsi, la jurisprudence administrative et constitutionnelle ont-elles fait évoluer le financement public des activités religieuses d'un régime marqué par l'exceptionnalité à un

21. Loi du 9 décembre 1905, art. 1: «La République assure la liberté de conscience. Elle garantit le libre exercice des cultes sous les seules restrictions édictées ci-après dans l'intérêt de l'ordre public. »

22. Constitution, art. 61-1.

23. Cons. const., déc. 21 févr. 2013, précit.

24. On ne développera pas ce point ici, malgré son importance. V. sur cette question "Les transformations coutumières de la loi de 1905 », notre contribution dans Droit et religion en Europe: Études en l'honneur de Francis Messner (Presses universitaires de Strasbourg, 2014, p. 519-540). On s'est attaché, dans cet article des Mélanges en l'honneur de Francis Messner, à expliciter les changements intervenus dans l'interprétation de la loi de 1905, à propos notamment du régime de l'affectation légale et de l'interdiction des financements publics de l'article 2. Ainsi qu'on a pu l'expliquer, ces changements résultent fondamentalement des évolutions de la sociologie religieuse et des rapports entre l'autorité publique et les religions. 
régime de droit commun de plus en plus homogène. En Alsace-Moselle et dans les territoires de l'outre-mer où la loi de 1905 n'a pas été introduite, le caractère religieux d'une activité ne suffit plus aujourd'hui à en justifier à lui seul le financement par les collectivités publiques. En régime de séparation au contraire, il ne suffit plus à lui seul à l'interdire. Dans l'un et l'autre cas, la légalité d'un financement public dépend maintenant de l'intérêt général à la réalisation duquel il contribue, et qui doit être dissocié de l'intérêt religieux particulier qu'il présente par ailleurs. De la sorte, le régime du financement public des activités religieuses tend à se rapprocher de celui des autres activités sociales prises en charge par les personnes privées, à cette différence près que l'intérêt public justifiant le financement ne peut résulter de l'activité religieuse elle-même, ce qui n'est pas le cas pour les autres activités sociales telles que la culture, l'art ou le sport. Enfin, les régimes historiques de financement obligatoire qui, en France, prennent la forme du salariat des ministres du culte, sont aujourd'hui fortement fragilisés, et même si le Conseil constitutionnel a choisi de renvoyer au législateur le soin d'y mettre fin, il est vraisemblable qu'ils ne survivront pas éternellement. Il y a une cohérence dans les transformations actuelles du régime financier des religions, et plutôt que d'en appeler toujours à la loi de 1905 et à son article 2, il faut accepter de voir cette cohérence pour comprendre et accompagner les évolutions qui se dessinent dans notre droit de la laïcité et des activités religieuses, et qui n'en sont qu'à leurs débuts. 